\title{
WAPP - Wideband Arecibo Pulsar Processor
}

\author{
A. Dowd, W. Sisk \\ Arecibo Observatory, HC3 Box 53995, Arecibo, PR 00612, USA \\ J. Hagen \\ Arecibo Observatory, HC3 Box 53995, Arecibo, PR 00612, USA \\ NRAO, $949 \mathrm{~N}$ Cherry Ave., Tucson, AZ 85721, USA
}

\begin{abstract}
The WAPP is a new Pulsar Processor for Arecibo Observatory. Key features are wideband operation $(100 \mathrm{MHz})$ and good time resolution (16 microseconds).
\end{abstract}

The WAPP is a new facility pulsar backend for Arecibo Observatory. The initial system will be available for observations starting in Feburary 2000. In its initial implementation, it will handle wideband pulsar observations with particular utility as a search machine or for single pulse work. This instrument represents the first operational stage of an on-going development program. The WAPP does incoherent pulsar detection. It uses a Linux computer to rapidly unload a hardware digital correlator. The resulting correlation functions are written to disk as 16 bit unsigned integers with minimal processing. The initial system will have two IF channels of up to $100 \mathrm{MHz}$ each. Nominally these channels will be configured for dual polarization observations. It is possible to measure autocorrelations from a single band or from both bands

\section{Key Points about system available in Feb 2000}

1. Wideband Operation: $100 \mathrm{MHz}$ and $50 \mathrm{MHz}$ modes

2. Up to $16 \mu$ s time resolution

3. Full Cross-Polarization Measurements

4. Search, Single Pulse modes

5. Incoherent (Correlation) Detection

6. 3 level and 9 level sampling modes

\section{Planned Upgrades to WAPP}

1. Flexible Bandwidth Selection from $100 \mathrm{MHz}$ down to $195.3 \mathrm{KHz}$

2. Spectra; Line Modes, Full 32 Bit Integration of correlation values

3. Timing Modes

4. Bandwidth expansion to $400 \mathrm{MHz}$ 


\section{References}

Xilouris, K., Salter C., Cordes J. M., Pulsar Science with the Upgraded Arecibo Telescope, Workshop Proceedings, 1996

\section{$100 \mathrm{MH}$ x 2 IF Pulsar Processor Module}

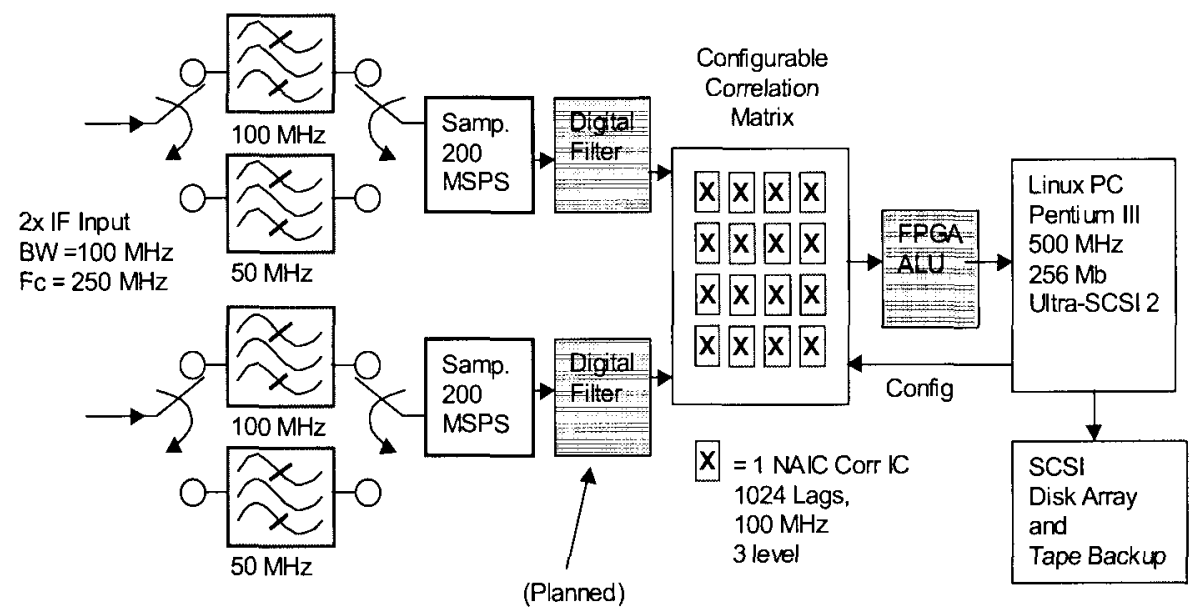

Table 1 - Available Modes for Searching or Single Pulse

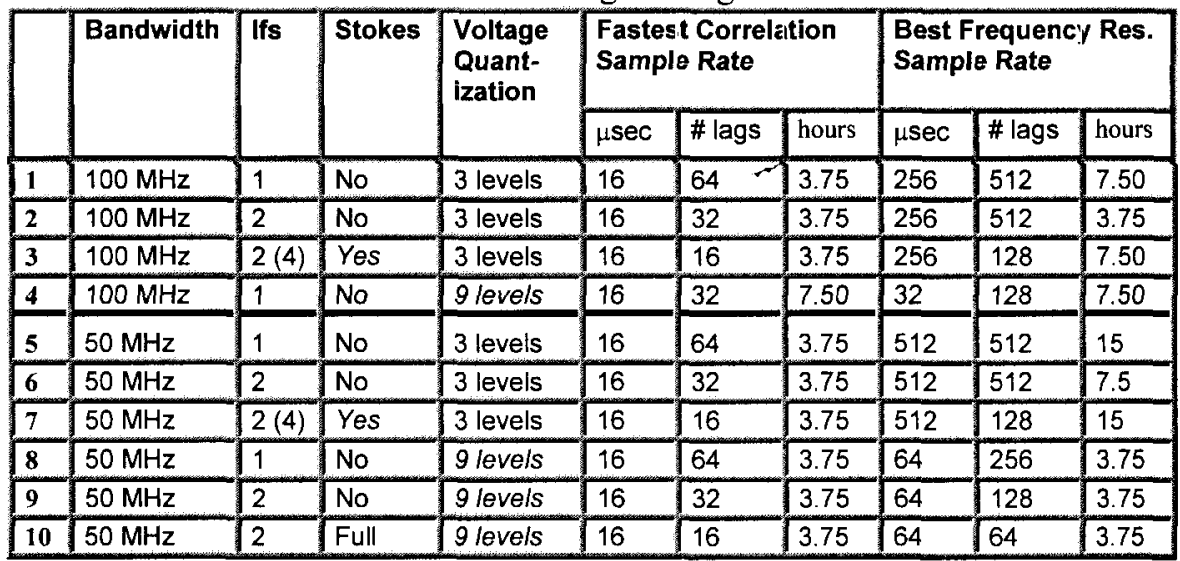

Hours - indicates how long WAPP can take data if the entire 108 Gbytes is used.

\# lags - indicates lags PER correlation measurement 